\title{
Validation of first chemistry mode retrieval results from the new limb-imaging FTS GLORIA with correlative MIPAS-STR observations
}

\author{
W. Woiwode ${ }^{1}$, O. Sumińska-Ebersoldt ${ }^{1}$, H. Oelhaf ${ }^{1}$, M. Höpfner ${ }^{1}$, G. V. Belyaev ${ }^{2}$, A. Ebersoldt ${ }^{3}$, F. Friedl-Vallon ${ }^{1}$, \\ J.-U. Grooß ${ }^{4}$, T. Gulde ${ }^{1}$, M. Kaufmann ${ }^{4}$, A. Kleinert ${ }^{1}$, M. Krämer ${ }^{4}$, E. Kretschmer ${ }^{1}$, T. Kulessa ${ }^{5}$, G. Maucher ${ }^{1}$, \\ T. Neubert ${ }^{6}$, C. Piesch ${ }^{1}$, P. Preusse ${ }^{4}$, M. Riese ${ }^{4}$, H. Rongen $^{6}$, C. Sartorius ${ }^{1}$, G. Schardt ${ }^{6}$, A. Schönfeld ${ }^{4}$, \\ D. Schuettemeyer ${ }^{7}$, M. K. Sha ${ }^{1}$, F. Stroh ${ }^{4}$, J. Ungermann ${ }^{4}$, C. M. Volk ${ }^{8}$, and J. Orphal ${ }^{1}$ \\ ${ }^{1}$ Institute of Meteorology and Climate Research, Karlsruhe Institute of Technology, Karlsruhe, Germany \\ ${ }^{2}$ Myasishchev Design Bureau, Zhukovsky-5, Moscow Region, Russia \\ ${ }^{3}$ Institute for Data Processing and Electronics, Karlsruhe Institute of Technology, Karlsruhe, Germany \\ ${ }^{4}$ Institute of Energy and Climate Research - Stratosphere (IEK-7), Forschungszentrum Jülich, Jülich, Germany \\ ${ }^{5}$ Central Institute for Engineering, Electronics and Analytics - Engineering and Technology (ZEA-1), \\ Forschungszentrum Jülich, Jülich, Germany \\ ${ }^{6}$ Central Institute for Engineering, Electronics and Analytics - Electronic Systems (ZEA-2), \\ Forschungszentrum Jülich, Jülich, Germany \\ ${ }^{7}$ European Space Agency, Mission Science Division, Noordwijk, the Netherlands \\ ${ }^{8}$ Department of Physics, University of Wuppertal, Wuppertal, Germany \\ Correspondence to: W. Woiwode (wolfgang.woiwode@kit.edu)
}

Received: 31 October 2014 - Published in Atmos. Meas. Tech. Discuss.: 18 December 2014

Revised: 24 April 2015 - Accepted: 12 May 2015 - Published: 19 June 2015

\begin{abstract}
We report first chemistry mode retrieval results from the new airborne limb-imaging infrared FTS (Fourier transform spectrometer) GLORIA (Gimballed Limb Observer for Radiance Imaging of the Atmosphere) and comparisons with observations by the conventional airborne limb-scanning infrared FTS MIPAS-STR (Michelson Interferometer for Passive Atmospheric Sounding STRatospheric aircraft). For GLORIA, the flights aboard the high-altitude research aircraft M55 Geophysica during the ESSenCe campaign (ESa Sounder Campaign 2011) were the very first in field deployment after several years of development. The simultaneous observations of GLORIA and MIPAS-STR during the flight on 16 December 2011 inside the polar vortex and under conditions of optically partially transparent polar stratospheric clouds (PSCs) provided us the first opportunity to compare the observations by two different infrared FTS generations directly. We validate the GLORIA results with MIPAS-STR based on the lower vertical resolution of MIPAS-STR and compare the vertical resolutions of the instruments derived from their averaging ker-
\end{abstract}

nels. The retrieval results of temperature, $\mathrm{HNO}_{3}, \mathrm{O}_{3}, \mathrm{H}_{2} \mathrm{O}$, CFC-11 and CFC-12 show reasonable agreement of GLORIA with MIPAS-STR and collocated in situ observations. For the horizontally binned hyperspectral limb images, the GLORIA sampling outnumbered the horizontal cross-track sampling of MIPAS-STR by up to 1 order of magnitude. Depending on the target parameter, typical vertical resolutions of 0.5 to $2.0 \mathrm{~km}$ were obtained for GLORIA and are typically a factor of 2 to 4 better compared to MIPAS-STR. While the improvement of the performance, characterization and data processing of GLORIA are the subject of ongoing work, the presented first results already demonstrate the considerable gain in sampling and vertical resolution achieved with GLORIA. 


\section{Introduction}

Passive infrared limb-emission observations from airborne, balloon-borne and space-borne platforms allow detailed observations of temperature, chemical composition and cloud properties in the upper troposphere and lower stratosphere (UTLS) region (Offermann et al., 1999; Fischer et al., 2008, and references therein). Observations by the airborne limbsounding FTS (Fourier transform spectrometer) MIPAS-STR (Michelson Interferometer for Passive Atmospheric Sounding - STRatospheric aircraft; Piesch et al., 1996) and the airborne limb-sounder CRISTA-NF (Cryogenic Infrared Spectrometers and Telescopes for the Atmosphere - New Frontiers; Kullmann et al., 2004) have shown that filamentary structures and clouds in the UTLS can be resolved with high horizontal and vertical resolution (Spang et al., 2008; Ungermann et al., 2012, 2013; Woiwode et al., 2012, and references therein).

We report retrieval results from observations by the new airborne limb-imaging FTS GLORIA (Gimballed Limb Observer for Radiance Imaging of the Atmosphere) (FriedlVallon et al., 2014; Riese et al., 2014, and references therein) associated with its first deployment in the field during the ESSenCe (ESa Sounder Campaign 2011) campaign. GLORIA performed its maiden flights aboard the high-altitude research aircraft Geophysica (for details about the Geophysica see Stefanutti et al., 1999) and probed the early Arctic polar vortex in December 2011 (Kaufmann et al., 2015). Although the GLORIA deployment during ESSenCe had a technical focus, several of the first observations show a high quality and allowed for accurate retrievals of atmospheric parameters.

GLORIA combines a Michelson interferometer with an imaging detector array, resulting in a much higher spatial sampling than can be achieved with conventional limbscanning single-pixel FTS. The GLORIA spectrometer has been operated in two different modes, each with different scientific aims: the chemistry mode observations are characterized by a high spectral resolution in combination with a medium (though considerably higher compared to conventional techniques) horizontal cross-track sampling, whereas the dynamics mode observations are characterized by a medium spectral and extremely high horizontal crosstrack sampling. The extremely dense dynamics mode observations include azimuth scanning and can be combined with dedicated flight patterns (e.g., hexagons) to enable tomographic retrievals. First results of GLORIA dynamics mode observations, including also tomographic retrievals, are reported by Kaufmann et al. (2015). The GLORIA chemistry mode observations reported here involved fixed pointing and aim at demonstrating the capabilities of classical 1-D retrievals of an extended set of trace gases enabled by the higher spectral resolution. An overview on the ESSenCe campaign and first GLORIA results are given by Kaufmann et al. (2013).
Here we present GLORIA chemistry mode retrieval results for the ESSenCe flight on 16 December 2011 and validate them with observations by the conventional limb-scanning FTS MIPAS-STR. Since both instruments observed the same air masses, direct comparisons of the retrieval results from both instruments of two different generations are possible. In Sects. 2 and 3, we briefly introduce GLORIA and MIPASSTR and compare the characteristics of the instruments. In Sect. 4, we discuss the meteorological conditions and sampling during the flight on 16 December 2011. In Sect. 5, we present comparisons between the GLORIA and MIPAS-STR as well as available collocated in situ observations, and our results are summarized in Sect. 6.

\section{Overview GLORIA}

GLORIA is a cryogenic limb-imaging FTS based on a cube corner Michelson linear slide interferometer and covers the spectral range from 780 to $1400 \mathrm{~cm}^{-1}$ within one single spectral channel. The instrument concept is introduced by Friedl-Vallon et al. (2014), and scientific objectives for GLORIA are discussed by Riese et al. (2014). Selected technical details and characteristics of GLORIA are summarized in Table 1. The GLORIA detector consists of a cryogenic $256 \times 256$ pixel $\mathrm{HgCdTe}$ large focal plane array. The spectrometer housing that contains the optical components is cooled to about $220 \mathrm{~K}$ to reduce instrumental background radiation. The spectrometer is mounted in a gimbal that allows for compensation of aircraft movements and for horizontal scanning in yaw direction from 45 to $135^{\circ}$ as well as for nadir observations. The observations discussed here were performed in limb mode pointing at $90^{\circ}$ in yaw direction (right side vs. aircraft nose, i.e., perpendicular to the aircraft true heading) and vertically fixed vs. the horizon.

During each interferometer sweep (i.e., hyperspectral image acquisition), DC-coupled interferograms are recorded for each individual pixel. GLORIA records double-sided interferograms. In the ESSenCe configuration, a subset of 128 vertical and 48 horizontal pixels was used for data acquisition. For the chemistry mode retrieval discussed in this context, the horizontal pixel rows were binned to reduce the measurement noise. A typical noise-equivalent spectral radiance (NESR) of $\sim 15 \times 10^{-9} \mathrm{~W} \mathrm{~cm}^{-2} \mathrm{sr}^{-1} \mathrm{~cm}$ was obtained for the binned spectra. For the ESSenCe chemistry mode observations, an optical path difference of $8 \mathrm{~cm}$ was employed. This corresponds to a spectral sampling of $0.0625 \mathrm{~cm}^{-1}$. After applying the Norton-Beer strong apodization, a spectral resolution of $0.121 \mathrm{~cm}^{-1}$ (full width at half maximum) was obtained (Norton and Beer, 1976). Each hyperspectral image covered a vertical angular range from about -3.6 to $0.4^{\circ}$ (horizontal view corresponds to $0^{\circ}$ ). For a typical flight altitude of $\sim 17 \mathrm{~km}$, this corresponds to a vertical coverage from $\sim 2 \mathrm{~km}$ to flight altitude. Additional vertical information was provided by the upward-viewing pixels. One hyperspectral 
Table 1. Comparison of instrumental and measurement characteristics between GLORIA and MIPAS-STR. Data for GLORIA correspond to chemistry mode observations in ESSenCe configuration (CM: chemistry mode).

\begin{tabular}{|c|c|c|}
\hline & GLORIA (CM) & MIPAS-STR \\
\hline Technique & limb-imaging FTS & limb-scanning FTS \\
\hline Spectral sampling & $0.0625 \mathrm{~cm}^{-1}$ & $0.0360 \mathrm{~cm}^{-1}$ \\
\hline Spectral range & 1 channel from 780 to $1400 \mathrm{~cm}^{-1}$ & 4 channels within 725 to $2100 \mathrm{~cm}^{-1}$ \\
\hline Detector & $256 \times 256$ pixel $\mathrm{HgCdTe}^{\mathrm{a}}$ & Si : As blocked-impurity-band \\
\hline Typical NESR & $\sim 15 \times 10^{-9} \mathrm{~W} \mathrm{~cm}^{-2} \mathrm{sr}^{-1} \mathrm{~cm}^{\mathrm{b}}$ & $\sim 10 \times 10^{-9} \mathrm{~W} \mathrm{~cm}^{-2} \mathrm{sr}^{-1} \mathrm{~cm}$ \\
\hline $\begin{array}{l}\text { Characteristic vertical } \\
\text { sampling (limb geometry) }\end{array}$ & $\sim 140 \mathrm{~m} @ 12 \mathrm{~km}^{\mathrm{c}}$ & $\sim 1 \mathrm{~km}$ \\
\hline $\begin{array}{l}\text { Typ. horizontal sampling } \\
\text { (cross-track) }\end{array}$ & 1 hyperspectral limb image $/ \sim 2.3 \mathrm{~km}$ & $1 \mathrm{limb} \mathrm{scan} / \sim 28 \mathrm{~km}^{\mathrm{c}}$ \\
\hline
\end{tabular}

a 128 vertical $\times 48$ horizontal pixels used in ESSenCe configuration. ${ }^{b}$ binned row of quality filtered pixels. ${ }^{c}$ corresponding to flight altitude of $\sim 16.8 \mathrm{~km}$.

image was recorded within $\sim 12.3 \mathrm{~s}$, resulting in a horizontal sampling of $\sim 2.3 \mathrm{~km}$ along flight track. The retrieval results shown in the following correspond to a quality-filtered subset of 68 chemistry mode hyperspectral images acquired in the interval between 14:30 and 14:50 UTC during the discussed flight.

The spectral and radiometric calibration of the GLORIA observations is discussed by Kleinert et al. (2014). The spectral calibration is performed by scaling the spectral axis with a pixel-dependent scaling factor. The scaling factor is determined from the spectral positions of selected atmospheric $\mathrm{CO}_{2}$ lines. The radiometric calibration includes a correction for detector nonlinearity and the determination of the radiometric offset and gain. The radiometric offset is determined from upward-viewing observations that contain only weak atmospheric signatures and are dominated by the instrumental background radiation. The radiometric gain is determined from in-flight blackbody measurements and considering the radiometric offset.

Filtering of cloud-affected observations was performed using the differential method introduced by Kleinert and Glatthor (2011). Clouds were identified using the second derivative of the vertical profiles of the integrated radiances within the spectral range from 800 to $949 \mathrm{~cm}^{-1}$. The tropospheric cloud threshold was set to pixels exceeding the mean value by 5 times the standard deviation. Observations corresponding to lower altitudes were excluded from the retrieval.

From the sets of horizontally binned spectra associated with individual hyperspectral images, vertical profiles of atmospheric parameters as well as instrumental parameters were retrieved by using the fast line-by-line forward model KOPRA (Karlsruhe Optimized and Precise Radiative transfer Algorithm; Stiller et al., 2002) and the inversion module KOPRAFIT (Höpfner et al., 2001). A constrained global fit was applied to the combination of the binned spectra of each individual hyperspectral limb image (i.e., cloud- and qualityfiltered vertical set out of 128 binned spectra). The issued atmospheric and instrumental parameters were combined into a vector and were determined by Newtonian iteration (von Clarmann et al., 2003, and references therein).

The iteration process minimized the residuals between the measured spectra and synthetic spectra associated with the applied initial guess atmosphere (Rodgers, 2000, p. 85). For the retrieval of the atmospheric parameter profiles, a firstorder regularization approach was applied (Tikhonov, 1963; Phillips, 1962), constraining the resulting profile towards the shape of a defined a priori profile in case of low information content in the measurements.

Pressure and temperature profiles from the ECMWF (European Centre for Medium-Range Weather Forecasts) reanalysis were used as initial guess profiles for the calculation of the synthetic spectra. The same ECMWF temperature profiles served as a priori profiles for the inversion of temperature. Initial guess and a priori profiles for the trace gas retrievals were taken from the Polar Winter Profiles for MIPAS (Remedios et al., 2007). $\mathrm{CO}_{2}$ profiles were updated for the Arctic winter 2011/12 involving simultaneous $\mathrm{CO}_{2}$ in situ observations by HAGAR (High-Altitude Gas AnalyzeR; Riediger et al., 2000; Werner et al., 2010) aboard the Gephysica and considering the Mauna Loa record (see http://www.esrl.noaa.gov/gmd/ccgg/trends/).

Retrieval parameters were a line-of-sight offset correction, temperature and the trace gas volume mixing ratios of $\mathrm{HNO}_{3}$, $\mathrm{O}_{3}, \mathrm{H}_{2} \mathrm{O}, \mathrm{CFC}-11$ and $\mathrm{CFC}-12$. The spectral microwindows applied for the GLORIA retrieval are listed in Table 2. Spectral line and cross-section data were taken from the MIPAS database (Flaud et al., 2006, and references therein). Additional retrieval parameters were spectral shift for each microwindow as well as radiometric offset, wavenumberindependent background continuum and volume mixing ratios of interfering species (in different combinations, depending on the target parameter). A vertical grid with a spacing of $0.25 \mathrm{~km}$ in the discussed vertical range was applied for the GLORIA retrievals. 
Table 2. Retrieval microwindows (MWs) used for GLORIA and MIPAS-STR.

\begin{tabular}{llll}
\hline Parameter & $\begin{array}{l}\text { MWs GLORIA } \\
{\left[\mathrm{cm}^{-1}\right]}\end{array}$ & $\begin{array}{l}\text { MWs MIPAS-STR } \\
{\left[\mathrm{cm}^{-1}\right]}\end{array}$ & Spectral Transition \\
\hline $\begin{array}{l}\text { LOS/temperature } \\
\text { (via CO } 2 \text { signatures) }\end{array}$ & $957.0-965.5$ & $955.6-958.5$ & $00011 \leftarrow 10001$ \\
$\mathrm{HNO}_{3}$ & $876.0-880.0$ & $810.1-813.1$ & $11101 \leftarrow 10002$ \\
$\mathrm{O}_{3}$ & $778.0-782.5$ & $786.0-870.0$ & v5- and 2v9-bands \\
& & $787.0-788.0$ & v2-band \\
$\mathrm{CFC}-11$ & $842.0-848.0$ & $842.5-848.0$ & v4-band \\
$\mathrm{CFC}-12$ & $915.0-925.0$ & $918.9-920.6$ & v6-band \\
& & $921.0-922.8$ & rotational transition \\
\hline
\end{tabular}

\section{Overview MIPAS-STR}

MIPAS-STR is a conventional cryogenic limb-scanning FTS using a double pendulum interferometer (a modified version of the Michelson interferometer; Fischer and Oelhaf, 1996) and four spectral channels in the range from 725 to $2100 \mathrm{~cm}^{-1}$. Technical details of MIPAS-STR, the calibration procedure and the retrieval are discussed by Piesch et al. (1996), Keim et al. (2008) and Woiwode et al. (2012). Selected technical details of MIPAS-STR and GLORIA are compared in Table 1. MIPAS-STR employs four cryogenic $\mathrm{Si}$ : As blocked-impurity-band detectors. The optics is dryice cooled to about $210 \mathrm{~K}$ to reduce background radiation. Double-sided interferograms are recorded with an optical path difference of $13.9 \mathrm{~cm}$. This corresponds to a spectral sampling of $0.0360 \mathrm{~cm}^{-1}$. After applying the NortonBeer strong apodization, a spectral resolution of $0.069 \mathrm{~cm}^{-1}$ (full width at half maximum) is obtained (Norton and Beer, 1976). For the retrievals discussed here, non-averaged channel 1 spectra $\left(725\right.$ to $990 \mathrm{~cm}^{-1}$ ) with a typical NESR of $\sim 10 \times 10^{-9} \mathrm{~W} \mathrm{~cm}^{-2} \mathrm{sr}^{-1} \mathrm{~cm}$ were employed.

The MIPAS-STR observations were performed in the limb-scanning mode. The complete sets of spectra associated with full limb scans were used for the retrievals of the atmospheric parameter profiles. The scanning sequence applied during the discussed ESSenCe flight employed limb views between $\sim 4 \mathrm{~km}$ and flight altitude in steps of $\sim 1 \mathrm{~km}$. Below $9 \mathrm{~km}$, the spacing increased towards $2 \mathrm{~km}$. Two additional upward-viewing elevation angles of 3 and $8^{\circ}$ were included in each sequence. One full limb scan was performed typically in $\sim 2.5 \mathrm{~min}$ and included atmospheric observations as well as blackbody and zenith view calibration measurements. For the flight leg of the discussed GLORIA observations, this corresponds to a horizontal distance along flight track of $\sim 28 \mathrm{~km}$. During the discussed flight MIPAS-STR performed comprehensive sampling, which was interrupted only a few times by aircraft maneuvers, electromagnetic disturbances (e.g., radio noise) and a dedicated measurement phase for detector nonlinearity characterization (16:00 to 16:12 UTC).
Cloud filtering was performed according to Spang et al. (2004). A rather weak cloud index threshold of 2.0 was applied, and partly cloud-affected observations were included in the retrieval. The retrieval approach applied for the MIPAS-STR observations and its validation are discussed by Woiwode et al. (2012). The same forward and inversion model, initial guess and a priori profiles as well as spectral line and cross-section data were used as for GLORIA. In Table 2, the spectral microwindows used for the MIPASSTR retrieval are compared with the microwindows used for the GLORIA retrieval. It should be noted that the GLORIA and MIPAS-STR observations were processed independently; therefore the retrieval setups differ from each other.

The MIPAS-STR retrieval was performed sequentially. First, temperature and trace gases with spectral signatures only weakly disturbed by spectral signatures of non-target gases were retrieved. Keeping the profiles of the previously retrieved species constant, gases with spectral signatures significantly affected by the previously determined gases were retrieved. In each retrieval step, spectral shift and wavenumber-independent background continuum were determined as additional parameters for each microwindow. No radiometric offset was retrieved. To better account for contributions from polar stratospheric clouds (PSCs) observed during this flight (see Sect. 4), wavenumber-independent background continuum was retrieved logarithmically. A vertical grid with a spacing of $0.5 \mathrm{~km}$ in the considered vertical range was applied for the MIPAS-STR retrievals.

\section{Campaign and meteorological situation}

The ESSenCe field campaign was based in Kiruna, Sweden $\left(68^{\circ} \mathrm{N} / 20^{\circ} \mathrm{E}\right)$. During the Geophysica flight on 16 December 2011, the polar vortex had already consolidated and showed low core temperatures allowing for the existence of PSCs. Figure 1 shows the flight path of the Geophysica together with the tangent points of the GLORIA and MIPASSTR observations. The entire flight was performed inside the polar vortex according to the definition of Nash et al. (1996) 


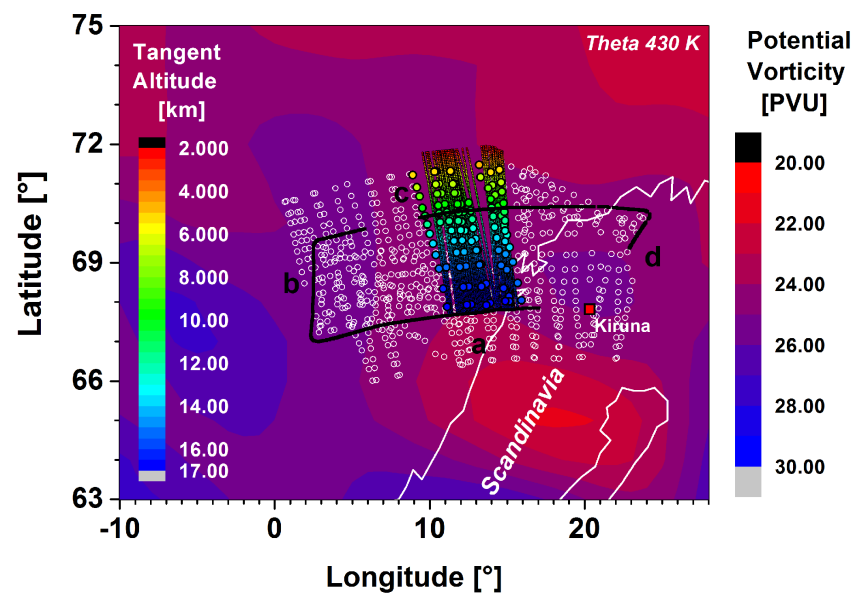

Figure 1. Flight path of the Geophysica (black line) during the flight on 16 December 2011. Tangent points of the MIPAS-STR observations during the entire flight (open white circles) and observations selected for comparison with GLORIA (solid circles, color-coded with altitude). Tangent points of GLORIA observations (solid dots, color-coded with altitude). Contour: potential vorticity at the potential temperature level of $430 \mathrm{~K}$ (approximately $18 \mathrm{~km}$ altitude).

(i.e., potential vorticity higher than 20 PVU at the potential temperature level of $430 \mathrm{~K}$ ). The flight was performed in a clockwise pattern, and GLORIA as well as MIPAS-STR pointed towards the center of the flight path. The dense pattern of the tangent points of the GLORIA observations indicates the considerable gain in sampling compared to MIPASSTR.

Figure 2 shows the vertical flight profile of the Geophysica together with the tangent points of the MIPAS-STR observations and the interpolated cloud index according to Spang et al. (2004). The GLORIA observations presented in the following were performed between 14:30 and 14:50 UTC during the end of the ascent phase and within the first flight phase at a constant altitude of $\sim 16.8 \mathrm{~km}$ (beginning of section a). Around 15:30 UTC, the Geophysica further climbed to the second phase at a constant altitude of $\sim 17.7 \mathrm{~km}$. The descent phase was entered after 17:00 UTC.

According to Spang et al. (2004), cloud index values higher than 4 indicate cloud-free conditions. Cloud index values close to 1 indicate strongly cloud-affected (i.e., optically thick) conditions, while values in between indicate partly cloud-affected scenarios. The interpolated vertical cross section of the MIPAS-STR cloud index shown in Figure 2 exhibits low values at all observed altitudes and during the entire flight. Cloud index values higher than 4 (i.e., cloud-free conditions) are found between flight altitude and $\sim 13 \mathrm{~km}$ at the beginning of flight section a, at the end of section $\mathrm{c}$ and in section d (i.e., in the eastern part of the flight; cf. Fig. 1). Except for the last limb scans in section c, also in these sections cloud index values lower than 4 are found for the observations directly below flight altitude. Cloud index values close

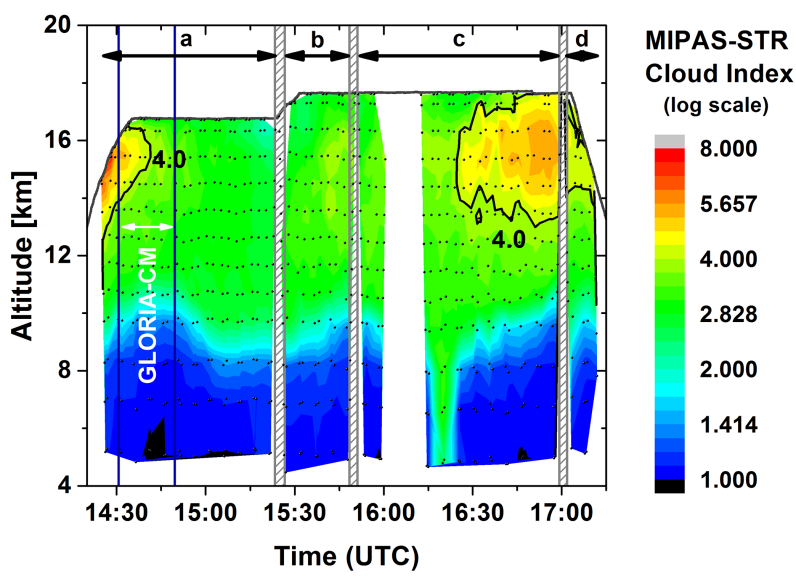

Figure 2. Vertical flight profile of the Geophysica (grey line), vertical distribution of the MIPAS-STR observations (black dots) and interpolated cloud index for the flight on 16 December 2011. Blue vertical lines indicate the time window of the presented GLORIA chemistry mode observations. Grey hatched areas indicate turns performed by the Geophysica between the different flight sections.

to one are approached at altitudes below 10 to $11 \mathrm{~km}$ and indicate the tropospheric cloud top. The only exception is the narrow time window around 16:20 UTC, showing partially transparent conditions down to altitudes below $5 \mathrm{~km}$.

During the entire flight, typical cloud index values around 2 are found at flight altitude. The low cloud index values indicate an extended partially transparent PSC. A weak minimum of the cloud index, corresponding to increased cloud influence, is located close to flight altitude around 15:20 UTC. Between about 14:40 and 16:30 UTC, no sharp boundary between low cloud index values around flight altitude and lower stratospheric altitudes can be identified. Since most of the observations were performed from inside the PSC, spectra corresponding to lower potentially cloud-free tangent altitudes are already cloud-affected. Therefore, it is likely that also in this time interval (approximately) cloud-free vertical sections were present between the PSC and the optically thick tropospheric clouds, while the lower boundary of the PSC is hardly or not at all resolved. Overall, lower cloud index threshold values may be considered for observations from inside a PSC and having their tangent points at lower altitudes depending on the local PSC scenario.

During this flight, MIPAS-STR and the Geophysica temperature sensor observed temperatures mostly below $198 \mathrm{~K}$ at flight altitude (see Sect. 5). The observed values are close to the existence temperature of nitric acid trihydrate (NAT), which is typically between about 194 and $198 \mathrm{~K}$ (depending on the actual partial pressures of $\mathrm{H}_{2} \mathrm{O}$ and $\mathrm{HNO}_{3}$; see $\mathrm{Pe}$ ter and Grooß, 2012, and references therein). Thus, the low cloud index values and the observed temperatures are compatible with a PSC containing NAT around flight altitude. 


\section{Comparisons between GLORIA, MIPAS-STR and collocated in situ observations}

Figure 3 shows the retrieval results for temperature $(T)$ and the volume mixing ratios (VMR) of $\mathrm{HNO}_{3}, \mathrm{O}_{3}, \mathrm{H}_{2} \mathrm{O}$, CFC11 and CFC-12 retrieved from GLORIA and MIPAS-STR together with collocated in situ observations aboard the Geophysica. The in situ temperature measurements were provided by the Geophysica UCSE (Unit for Connection with Scientific Equipment) temperature sensor. In situ measurements of total water were provided by FISH (Fast In situ Stratospheric Hygrometer; Zöger et al., 1999). The in situ observations of CFC-11 and CFC-12 were provided by HAGAR (Riediger et al., 2000; Werner et al., 2010). The accuracy of the FISH $\mathrm{H}_{2} \mathrm{O}$ observations is $0.2 \mathrm{ppm}$ and for the HAGAR CFC-11 and CFC-12 observations 0.6 and $0.3 \%$, respectively (von Hobe et al., 2013, and references therein). The estimated error of the Geophysica temperature sensor is $2 \mathrm{~K}$ between 10 and $20 \mathrm{~km}$ altitude. It has to be kept in mind that the in situ observations were restricted to the flight trajectory, while GLORIA and MIPAS-STR integrated radiation along their viewing direction. But due to the fact that both the in situ observations and the remote sensing observations were located inside the polar vortex (cf. Fig. 1) and due to the close match between the observations, meaningful comparisons are possible here.

Depending on the target parameter, 39 to 42 vertical profiles were retrieved successfully from the 68 GLORIA hyperspectral limb images. From MIPAS-STR, nine vertical profiles were retrieved successfully from nine limb scans in approximately the same time interval. Accordingly, a factor of $\sim 4.5$ more profiles were provided by GLORIA compared to MIPAS-STR in the considered time interval (locally by up to a factor of $\sim 8$ ).

In the left panels of Fig. 3a-f, all individual profiles from GLORIA and MIPAS-STR in the selected time interval are presented for the different target parameters. The shown in situ observations include all data points recorded during the entire flight from the ascent to the descent phase. Altitudes higher than the approximate flight level of the Geophysica in the time interval of the GLORIA observations (mostly $\sim 16.8 \mathrm{~km}$ ) are shaded in grey. At these altitudes, only very limited vertical information is obtained from the limb observations. Furthermore, the retrieval in this range is very sensitive to calibration uncertainties due to the weak spectral signatures observed under high viewing angles. For MIPASSTR, the retrieval results at flight altitude and directly above are still relatively reliable due to a higher spectral resolution and a very accurate radiometric offset calibration using zenith view observations. For GLORIA the resulting uncertainties are higher due to the lower spectral resolution and higher calibration uncertainties.

The middle panels in Fig. 3a-f show the residuals between the mean profiles of GLORIA and MIPAS-STR as well as the residuals between the mean profiles of GLORIA and the in situ profiles. For the calculation of the residual profiles between GLORIA and MIPAS-STR, all GLORIA profiles of the considered parameter were averaged. The resulting average profile was smoothed with the mean averaging kernel matrix of the corresponding MIPAS-STR profiles according to Rodgers (2000, p. 46-47). The residual profile between the smoothed GLORIA mean profile and the corresponding MIPAS-STR mean profile was calculated on the MIPASSTR retrieval grid.

For the calculation of the residuals between GLORIA and the in situ observations, all available in situ data points were sorted by altitude. The dense observations by the Geophysica temperature sensor and the dense $\mathrm{H}_{2} \mathrm{O}$ observations by FISH were furthermore subjected to a 10-times running mean in order to minimize biases from local atmospheric fine structures or outliers. All in situ observations were interpolated to the GLORIA retrieval grid, and the corresponding residuals were calculated using the unsmoothed mean profiles from GLORIA.

In the right panels of Fig. 3a-f, the vertical resolutions of the individual profiles retrieved from GLORIA and MIPASSTR are shown. The vertical resolutions were calculated using the diagonal elements of the averaging kernel matrices according to Purser and Huang (1993).

The comparison of the retrieved vertical profiles of temperature (Fig. 3a) shows that the GLORIA profiles mostly scatter within a few kelvin around the MIPAS-STR profiles and the in situ observations. The higher scattering in the GLORIA observations might indicate small-scale temperature variations not covered by the in situ measurements and not resolved by MIPAS-STR. Another explanation might be oscillations in the GLORIA retrieval results (e.g., due to variable calibration uncertainties or a weak retrieval constraint). The latter is supported by the fact that the MIPAS-STR profiles and the in situ observations are very consistent and more compact.

The absolute residual values between the GLORIA and MIPAS-STR mean profiles are within $\pm 2 \mathrm{~K}$ below flight altitude. For GLORIA, systematically lower temperatures are found below about $15 \mathrm{~km}$, and higher temperatures above. Except for differences between about 12 and $13.5 \mathrm{~km}$ as well as at $16.5 \mathrm{~km}$ and above, the residual values between GLORIA and the Geophysica temperature sensor show a similar pattern. For the temperature profiles retrieved from GLORIA, a typical vertical resolution of about 0.5 to $1.0 \mathrm{~km}$ is obtained below flight altitude. Depending on the altitude, this is typically a factor of 2 to 4 higher compared to MIPAS-STR (typically around $2.0 \mathrm{~km}$ ).

The vertical profiles of $\mathrm{HNO}_{3}$ (Fig. 3b) retrieved from GLORIA and MIPAS-STR each show a compact pattern. The overall shapes of the $\mathrm{HNO}_{3}$ profiles obtained from MIPAS-STR are reproduced to a satisfying degree by GLORIA. The GLORIA mean profile shows systematically lower values by up to $1 \mathrm{ppbv}$ above $13.0 \mathrm{~km}$ compared to MIPASSTR. The maximum relative residual value of approximately 
(a)
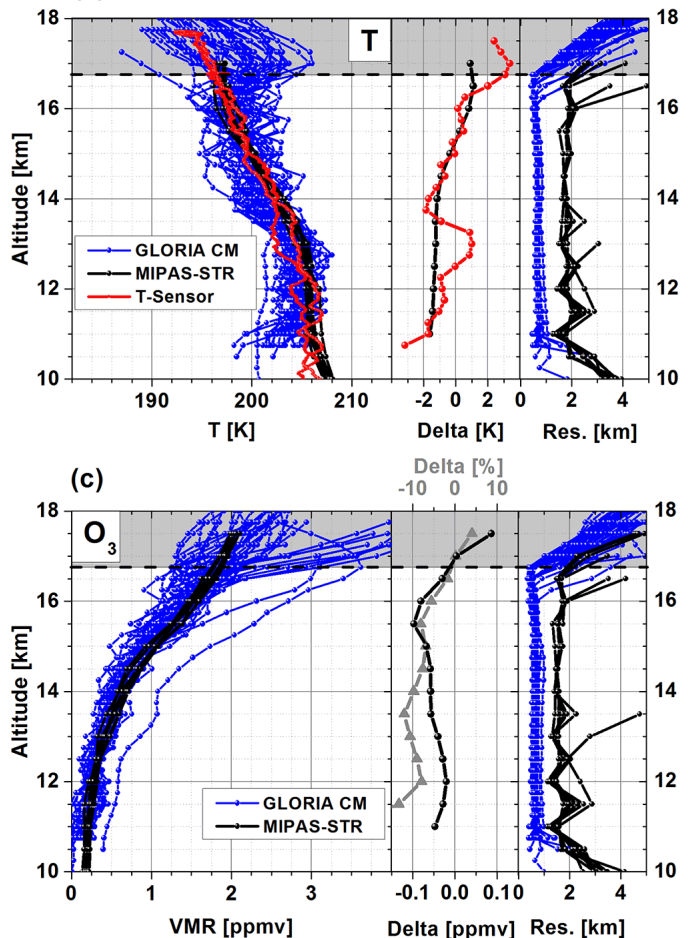

(e)

$\begin{array}{ccc}\text { Delta [\%] } \\ -15 \quad 0 & 15\end{array}$

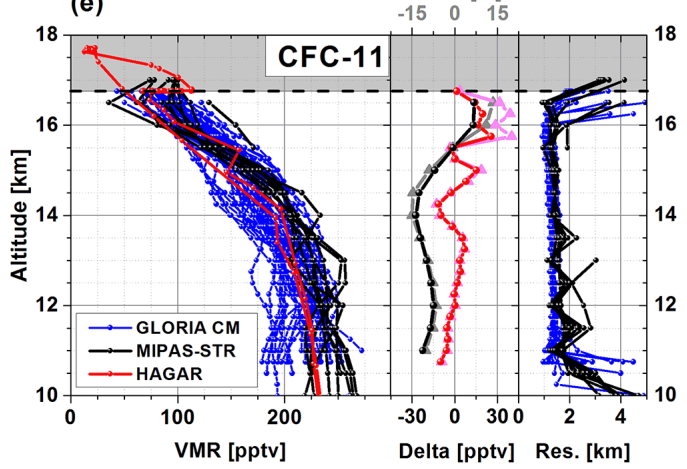

(b)

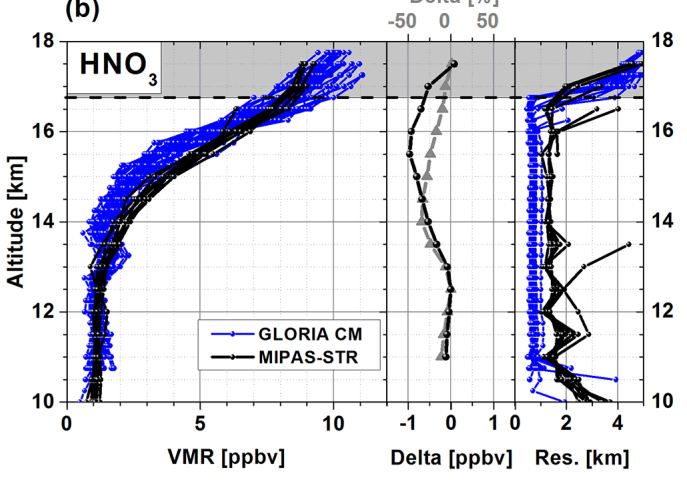

(d)

Delta [\%]
$-20 \quad 0 \quad 20$

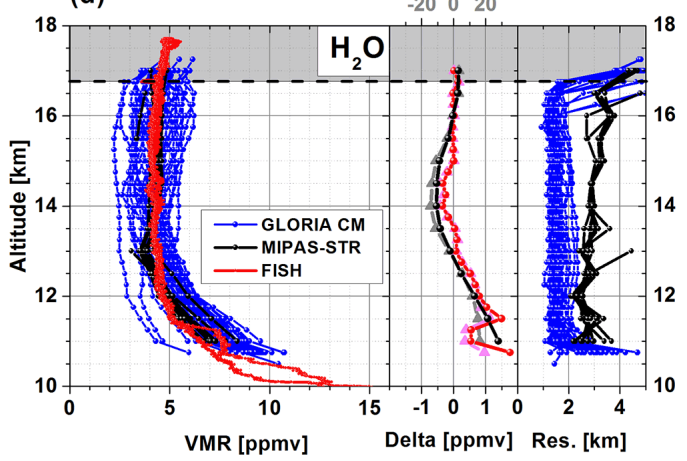

Delta [\%]

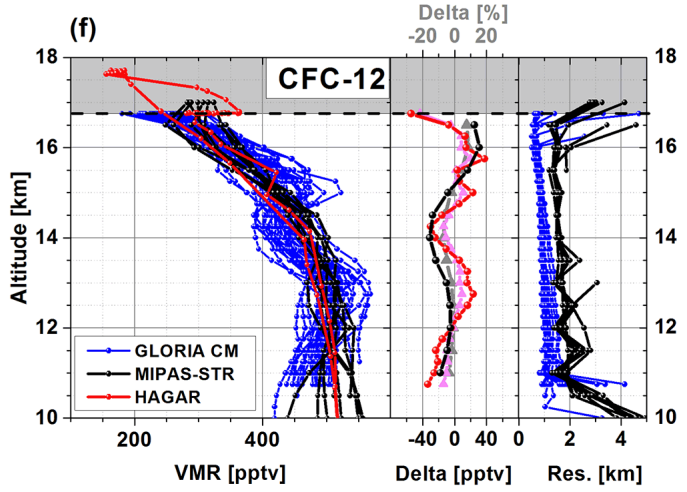

Figure 3. Comparison of retrieval results from GLORIA with MIPAS-STR and collocated in situ observations. Left panels: retrieved vertical profiles of indicated parameters from GLORIA (blue) and MIPAS-STR (black) together with available in situ profiles (red). Middle panels: absolute (black) and relative (grey) residuals between the mean profiles of GLORIA and MIPAS-STR as well as absolute (red) and relative (pink) residuals between mean profiles of GLORIA and the in situ observations. Right panels: vertical resolutions of the GLORIA (blue) and MIPAS-STR (black) profiles. Dashed horizontal black lines in all panels: approximate flight altitude during the GLORIA observations.

$-30 \%$ at $14 \mathrm{~km}$ is still relatively small in the context of the steep gradient in the $\mathrm{HNO}_{3}$ mixing ratios and the low absolute mixing ratios. The GLORIA observations furthermore show consistently a local maximum peaking at $13.25 \mathrm{~km}$, which is attributed to a vertical fine structure not resolved by MIPAS-STR. The vertical resolution of the GLORIA profiles is typically 0.5 to $1.0 \mathrm{~km}$ and a factor of $\sim 2$ higher compared to MIPAS-STR (often slightly better than $\sim 1.5 \mathrm{~km}$, coarser resolution towards lower altitudes).

The $\mathrm{O}_{3}$ profiles (Fig. 3c) retrieved from GLORIA show on average slightly lower values than MIPAS-STR below flight altitude. The residual of the mean profiles indicates small systematic differences of less than $-0.1 \mathrm{ppmv}$ and mostly within $-10 \%$. Beside the good agreement for $\mathrm{O}_{3}$, the residual values indicate a weak systematic bias of the GLORIA profiles towards lower mixing ratios. The typical vertical resolution obtained for GLORIA is between 0.5 and $1.0 \mathrm{~km}$, which is a factor of $\sim 3$ higher than for the MIPAS-STR results (slightly coarser than $\sim 1.5 \mathrm{~km}$, coarser resolution towards lower altitudes).

While the $\mathrm{H}_{2} \mathrm{O}$ profiles (Fig. 3d) retrieved from GLORIA show a significantly higher scattering compared to MIPAS- 
(a)

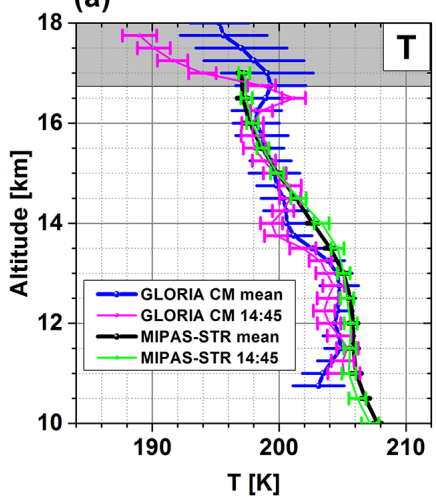

(d)

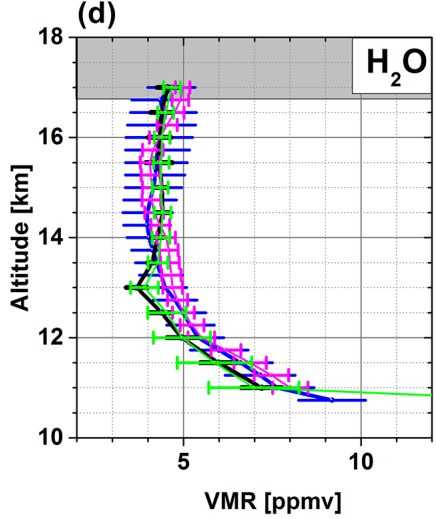

(b)

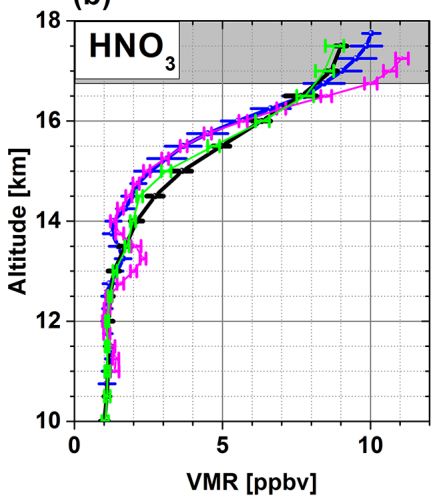

(e)

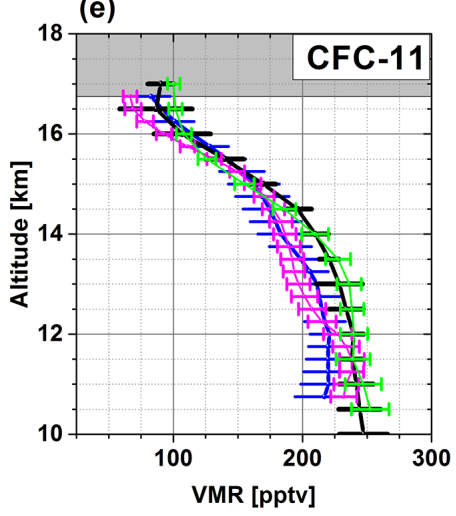

(c)
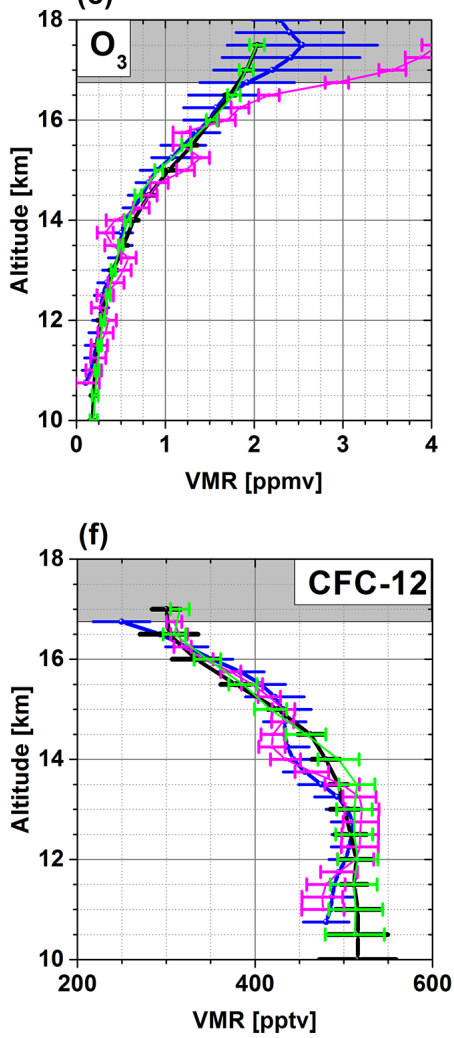

Figure 4. Mean and standard deviation of the GLORIA profiles (blue) and MIPAS-STR profiles (black) shown in Fig. 3. Individual nonaveraged profiles at $\sim 14: 45$ UTC together with random/variable uncertainties for GLORIA (magenta) and MIPAS-STR (green).

STR and the in situ observations, the shapes of the retrieved profiles mostly agree well with the collocated observations. Except for altitudes below $11.5 \mathrm{~km}$, the residuals between the GLORIA and MIPAS-STR mean profiles as well as between the GLORIA mean profile and FISH are within \pm 1 ppmv and $\pm 20 \%$. Compared to MIPAS-STR, systematically lower mixing ratios are indicated by GLORIA between 13 and $16 \mathrm{~km}$, and higher mixing ratios are found below. Compared to FISH, only slightly lower mixing ratios are found around $14 \mathrm{~km}$, while higher mixing ratios are found below $13 \mathrm{~km}$. For GLORIA, typical vertical resolutions around $1.0 \mathrm{~km}$ to slightly coarser than $2.0 \mathrm{~km}$ were obtained, which are a factor of 2 to 3 higher compared to MIPAS-STR (typically around 2.5 to $3.5 \mathrm{~km}$ ).

The profiles of CFC-11 (Fig. 3e) retrieved from GLORIA are in good agreement with the MIPAS-STR and HAGAR observations. The absolute residuals of the GLORIA mean profile compared to MIPAS-STR and HAGAR are within \pm 30 pptv and the relative residuals mostly within $\pm 15 \%$. While the agreement between GLORIA and HAGAR is better, the residual between GLORIA and MIPASSTR exhibits systematically lower mixing ratios for GLORIA below $15 \mathrm{~km}$, and systematically higher mixing ratios are found above. Except for the lower parts of the profiles where the MIPAS-STR vertical resolution becomes coarser, similar vertical resolutions around $1.5 \mathrm{~km}$ are obtained for GLORIA and MIPAS-STR. This is attributed to the higher spectral resolution and lower NESR of MIPAS-STR, allowing for a better exploitation of the weak CFC-11 signatures observed under the cold conditions of this flight.

The profiles of CFC-12 (Fig. 3f) show good agreement of GLORIA with MIPAS-STR and HAGAR. Below flight altitude, the absolute residuals for the mean profiles are within \pm 40 pptv and the relative residuals mostly within $\pm 10 \%$. Compared to MIPAS-STR, systematically lower mixing ratios are found below $\sim 15 \mathrm{~km}$ and higher mixing ratios above. The absolute and relative residual profiles between GLORIA and HAGAR are more variable with altitude and show small positive and negative values. Typical vertical resolutions of about 0.5 to $1.5 \mathrm{~km}$ are obtained for GLORIA, which is a factor of $\sim 2$ higher compared to MIPAS-STR (typically about 1.5 to $2.5 \mathrm{~km}$ ).

In Fig. 4, the corresponding mean profiles derived from GLORIA and MIPAS-STR are shown together with their standard deviations. Also shown are selected individual profiles of both instruments, measured at $\sim 14$ : 45 UTC. Errors bars of the individual GLORIA profiles include the following random/variable error components: (i) spectral noise er- 
ror (from noise covariance matrix), (ii) radiometric gain error (effect on retrieval result from $2 \%$ gain modification) and (iii) line-of-sight error (effect on retrieval result from lineof-sight modification of $0.7 \mathrm{arcmin}$ ). The error components were treated as $1 \sigma$ uncertainties and combined by the root of the square sum. Radiometric gain and line-of-sight errors are estimates based on the state of the GLORIA characterization related to ESSenCe. For MIPAS-STR, the same error budget was performed, with the only exception that for estimation of the line-of-sight error all elevation angles of the limb sequence were modified more conservatively by $1 \mathrm{arcmin}$. Spectral line data and cross-section errors were not considered, since the same spectral database and similar spectral microwindows were used for the MIPAS-STR and GLORIA retrievals. Thus, these errors are expected to cancel out in the comparisons between GLORIA and MIPAS-STR.

The mean profiles from GLORIA and MIPAS-STR agree mostly within their standard deviations. Their differences reflect the characteristics of the residual profiles between the smoothed GLORIA mean profiles and the MIPAS-STR mean profiles discussed in the context of Fig. 3. For $\mathrm{HNO}_{3}$, the differences between the GLORIA and MIPAS-STR mean profiles exceed the sum of the standard deviations between 13.5 and $16 \mathrm{~km}$ and hint at more pronounced systematic errors.

While the mean and individual GLORIA and MIPASSTR profiles shown in Fig. 4 have similar overall shape and comparable absolute values, the individual GLORIA profiles show a higher variability. The vertical sections where the differences of the individual profiles exceed the sum of the GLORIA and MIPAS-STR error bars have extensions of $0.5 \mathrm{~km}$ to a few kilometers. The vertical structures in the GLORIA profiles often exceed the amplitudes of the GLORIA error bars (and therefore the noise error) and hint at variability of the atmospheric scenery. Contributions from nonidentified error sources however cannot be excluded. Horizontal variability of the atmospheric scenery along flight track also might play a role here, since the GLORIA hyperspectral image resulting in the shown vertical profile was recorded on a significantly shorter section of the flight track compared to the corresponding MIPAS-STR limb scan.

For $\mathrm{HNO}_{3}$, the mean profiles from GLORIA and MIPASSTR are in close agreement below $13.75 \mathrm{~km}$, while a significant systematic bias is observed above. Both the GLORIA mean profile and, to a higher extend, the individual profile show a local maximum around $13.25 \mathrm{~km}$. The maximum in the individual GLORIA profile exceeds the MIPAS-STR mean and individual profile as well as the GLORIA mean profile. Since the amplitude of the maximum significantly exceeds the GLORIA error bars, this structure can be interpreted as a structure in the vertical distribution of $\mathrm{HNO}_{3}$. Overall, the vertical extensions of the structures in the individual GLORIA profiles shown in Figs. 3 and 4 are mostly plausible given their associated vertical resolution and error bars. Many of the observed structures hint at variability in the atmospheric scenery.
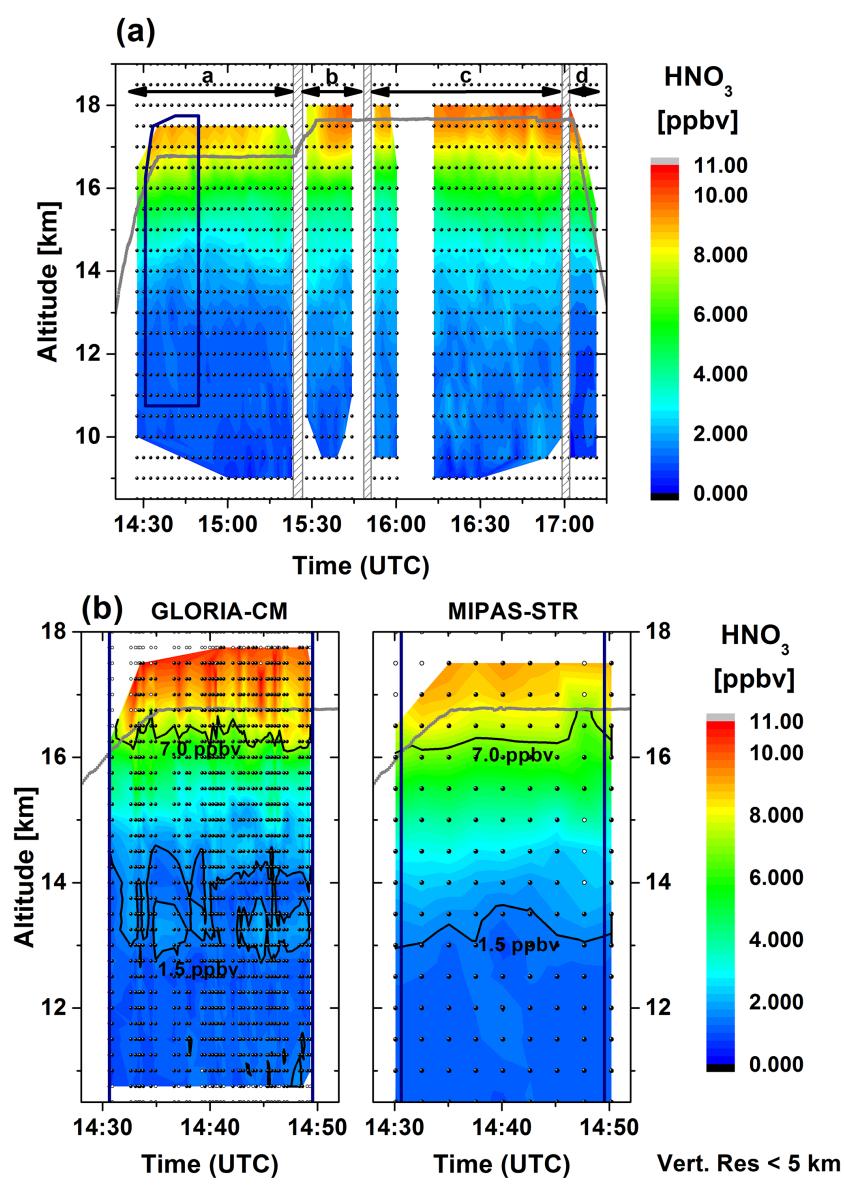

Figure 5. (a) Interpolated vertical cross section of $\mathrm{HNO}_{3}$ retrieved from MIPAS-STR for the entire flight. Grey hatched areas indicate turns performed by the Geophysica between the different flight sections. (b) $\mathrm{HNO}_{3}$ cross section retrieved from GLORIA and corresponding subsection of MIPAS-STR cross section. Black dots in all panels: used retrieval grids. Open black dots in lower panels: grid points excluded from interpolation due to low vertical resolution. Solid grey lines in all panels: flight altitude of the Geophysica.

In Fig. 5, we present vertical cross sections of $\mathrm{HNO}_{3}$ retrieved from GLORIA and MIPAS-STR. Figure 5a shows the $\mathrm{HNO}_{3}$ cross section retrieved from the MIPAS-STR observations during the entire flight. The black dots represent the retrieval grid applied for MIPAS-STR. The horizontal spacing indicates the horizontal sampling. The cross section shows a relatively homogenous $\mathrm{HNO}_{3}$ distribution along the flight track and the absence of prominent filamentary structures. In the region around the first turn (around 15:25 UTC), lower mixing ratios of $\mathrm{HNO}_{3}$ are found around flight altitude when compared to the other flight sections and hint at a dynamical structure.

The blue box indicates the region corresponding to the vertical $\mathrm{HNO}_{3}$ cross section that was retrieved from GLORIA (left panel in Fig. 5b), which is shown together with the corresponding subsection from MIPAS-STR below (right 
panel in Fig. 5b). Both GLORIA and MIPAS-STR show the steady overall increase of the $\mathrm{HNO}_{3}$ volume mixing ratios towards flight altitude. While the MIPAS-STR observations show a relatively smooth $\mathrm{HNO}_{3}$ distribution in the whole vertical range, the GLORIA cross section shows some weak fine structures between $\sim 12.5 \mathrm{~km}$ and flight altitude that hint at patterns not resolved by MIPAS-STR. The faint maxima located in the GLORIA cross section around $13.25 \mathrm{~km}$ are also identified in the corresponding vertical profiles in Figs. $3 \mathrm{~b}$ and $4 b$.

\section{Conclusions}

The Geophysica aircraft campaign in December 2011 provided us with the first opportunity to directly compare observations by two infrared FTS generations: the new limbimaging FTS GLORIA and the conventional limb-scanning FTS MIPAS-STR. During the discussed flight phase on 16 December 2011, the achieved horizontal cross-track sampling of the GLORIA chemistry mode hyperspectral limb images was by up to a factor of $\sim 11$ higher compared to the MIPAS-STR limb scans. From the presented GLORIA observations, locally up to a factor of $\sim 8$ more vertical profiles were retrieved successfully compared to MIPAS-STR in the same time step. The first GLORIA chemistry mode retrieval results show reasonable agreement with those from MIPASSTR and collocated in situ observations, bearing in mind that this was the very first deployment of a complex new instrument developed over several years.

The low cloud index values of the MIPAS-STR observations and the low temperatures observed by MIPAS-STR as well as the Geophysica temperature sensor indicate the presence of an optically partially transparent PSC. GLORIA reproduces the overall shapes of the vertical profiles determined from MIPAS-STR and the in situ observations well. Below flight altitude, the mean residuals between GLORIA and MIPAS-STR as well as GLORIA and the in situ observations are mostly within $\pm 2 \mathrm{~K}$ for temperature and within a few ten percent for trace gas volume mixing ratios. Systematic differences found between the retrieval results from GLORIA and MIPAS-STR hint at calibration uncertainties in the presented GLORIA data (e.g., systematic uncertainties in radiometric calibration and line-of-sight knowledge). Further improvement of the performance, characterization and calibration of the GLORIA observations from subsequent campaigns is the subject of ongoing work.

The significantly higher scattering in the GLORIA profiles compared to MIPAS-STR and the in situ observations is attributed to random uncertainties in the GLORIA observations (e.g., spectral noise, variable radiometric uncertainties or short-time line-of-sight variations) and might also indicate random-like fine structures. Faint and vertically narrow maxima and minima in the GLORIA results for $\mathrm{HNO}_{3}$ that extend over several profiles are attributed to fine structures not resolved by MIPAS-STR. Dependent on the retrieval parameter, typical vertical resolutions of 0.5 to $2.0 \mathrm{~km}$ are obtained for the profiles retrieved from the GLORIA observations. This is typically a factor of 2 to 4 higher compared to MIPAS-STR.

The presented results confirm the good performance of GLORIA during its very first airborne deployment and indicate potential for further improvements in both hardware and data processing. Dedicated upgrades in the instrument setup as well as the calibration and characterization procedure have meanwhile led to significant improvements in instrument performance and data quality in subsequent campaigns. A further simultaneous airborne deployment of the two instruments would thus be extremely helpful to check the behavior of the consolidated GLORIA instrument over a longer flight time and under variable atmospheric conditions.

The GLORIA observations presented here and more extensive atmospheric measurements during recent High Altitude and LOng Range Research Aircraft (HALO) campaigns (Riese et al., 2014) demonstrate the high gain in information accessible by the limb-imaging technique.

Acknowledgements. This work was supported by the European Space Agency/Mission Science Division under the ESSenCe project (Technical Assistance for the Deployment of Airborne Limbsounders during ESSenCe). We thank the ESSenCe coordination team and Myasishchev Design Bureau for providing the basis for a successful aircraft campaign. We thank Johannes Wintel (University of Wuppertal) for processing the HAGAR observations. ECMWF data for the GLORIA and MIPAS-STR retrievals were obtained via NILU's NADIR database. We acknowledge support by the Deutsche Forschungsgemeinschaft and Open Access Publishing Fund of the Karlsruhe Institute of Technology.

The article processing charges for this open-access publication were covered by a Research Centre of the Helmholtz Association.

Edited by: C. von Savigny

\section{References}

Fischer, H. and Oelhaf, H.: Remote Sensing of vertical profiles of atmospheric trace constitutents with MIPAS limb-emission spectrometers, Appl. Optics, 35, 2787-2796, 1996.

Fischer, H., Birk, M., Blom, C., Carli, B., Carlotti, M., von Clarmann, T., Delbouille, L., Dudhia, A., Ehhalt, D., Endemann, M., Flaud, J. M., Gessner, R., Kleinert, A., Koopman, R., Langen, J., López-Puertas, M., Mosner, P., Nett, H., Oelhaf, H., Perron, G., Remedios, J., Ridolfi, M., Stiller, G., and Zander, R.: MIPAS: an instrument for atmospheric and climate research, Atmos. Chem. Phys., 8, 2151-2188, doi:10.5194/acp-8-2151-2008, 2008.

Flaud, J.-M., Brizzi, G., Carlotti, M., Perrin, A., and Ridolfi, M.: MIPAS database: Validation of $\mathrm{HNO}_{3}$ line parameters using MI- 
PAS satellite measurements, Atmos. Chem. Phys., 6, 5037-5048, doi:10.5194/acp-6-5037-2006, 2006.

Friedl-Vallon, F., Gulde, T., Hase, F., Kleinert, A., Kulessa, T., Maucher, G., Neubert, T., Olschewski, F., Piesch, C., Preusse, P., Rongen, H., Sartorius, C., Schneider, H., Schönfeld, A., Tan, V., Bayer, N., Blank, J., Dapp, R., Ebersoldt, A., Fischer, H., Graf, F., Guggenmoser, T., Höpfner, M., Kaufmann, M., Kretschmer, E., Latzko, T., Nordmeyer, H., Oelhaf, H., Orphal, J., Riese, M., Schardt, G., Schillings, J., Sha, M. K., Suminska-Ebersoldt, O., and Ungermann, J.: Instrument concept of the imaging Fourier transform spectrometer GLORIA, Atmos. Meas. Tech., 7, 35653577, doi:10.5194/amt-7-3565-2014, 2014.

Höpfner, M., Blom, C. E., Echle, G., Glatthor, N., Hase, F., and Stiller, G.: Retrieval simulations for MIPAS-STR measurements, edited by: Smith, W. L., IRS 2000: Current Problems in Atmospheric Radiation; Proc. of the Internat. Radiation Symp., St. Petersburg, Russia, 24-29 July 2000, DEEPAK Publ., Hampton, Va., http://www.imk-asf.kit.edu/downloads/ffb/ IRS2000_proceedings_hoepfner_1.pdf, 2001.

Kaufmann, M., Blank, J., Friedl-Vallon, F., Gerber, D., Guggenmoser, T., Hoepfner, M., Kleinert, A., Sha, M. K., Oelhaf, H., Riese, M., Suminska-Ebersoldt, O., Woiwode, W., Siddans, R., Kerridge, B., Moyna, B., Rea, S., and Oldfield, M.: Technical assistance for the deployment of airborne limbsounders during ESSenCe, Technical report, European Space Agency, https://earth.esa.int/documents/10174/ 134665/ESSenCe_Final_Report, 2013.

Kaufmann, M., Blank, J., Guggenmoser, T., Ungermann, J., Engel, A., Ern, M., Friedl-Vallon, F., Gerber, D., Grooß, J. U., Guenther, G., Höpfner, M., Kleinert, A., Kretschmer, E., Latzko, Th., Maucher, G., Neubert, T., Nordmeyer, H., Oelhaf, H., Olschewski, F., Orphal, J., Preusse, P., Schlager, H., Schneider, H., Schuettemeyer, D., Stroh, F., Suminska-Ebersoldt, O., Vogel, B., M. Volk, C., Woiwode, W., and Riese, M.: Retrieval of threedimensional small-scale structures in upper-tropospheric/lowerstratospheric composition as measured by GLORIA, Atmos. Meas. Tech., 8, 81-95, doi:10.5194/amt-8-81-2015, 2015.

Keim, C., Liu, G. Y., Blom, C. E., Fischer, H., Gulde, T., Höpfner, M., Piesch, C., Ravegnani, F., Roiger, A., Schlager, H., and Sitnikov, N.: Vertical profile of peroxyacetyl nitrate (PAN) from MIPAS-STR measurements over Brazil in February 2005 and its contribution to tropical UT NOy partitioning, Atmos. Chem. Phys., 8, 4891-4902, doi:10.5194/acp-8-4891-2008, 2008.

Kleinert, A. and Glatthor, N.: Cloud detection for onboard IRLS co-addition, Technical Note, PREMIER - Consolidation of Requirements and Synergistic Retrieval Algorithms, Task 6.2 - WP 6220, Version 6, 2011

Kleinert, A., Friedl-Vallon, F., Guggenmoser, T., Höpfner, M., Neubert, T., Ribalda, R., Sha, M. K., Ungermann, J., Blank, J., Ebersoldt, A., Kretschmer, E., Latzko, T., Oelhaf, H., Olschewski, F., and Preusse, P.: Level 0 to 1 processing of the imaging Fourier transform spectrometer GLORIA: generation of radiometrically and spectrally calibrated spectra, Atmos. Meas. Tech., 7, 41674184, doi:10.5194/amt-7-4167-2014, 2014.

Kullmann, A., Riese, M., Olschewski, F., Stroh, F., and Grossmann, K. U.: Cryogenic infrared spectrometers and telescopes for the atmosphere - new frontiers, Proc. SPIE, 5570, 423-432, 2004.
Nash, E. R., Newman, P. A., Rosenfield, J. E., and Schoeberl, M. R.: An objective determination of the polar vortex using Ertel's potential vorticity, J. Geophys. Res., 101, 9471-9478, 1996.

Norton, H. and Beer, R.: New apodizing functions for Fourier spectrometry, J. Opt. Soc. Am., 66, 259-264, (Errata J. Opt. Soc. Am., 67, 419, 1977), 1976.

Offermann, D., Grossmann, K.-U., Barthol, P., Knieling, P., Riese, M., and Trant, R.: Cryogenic Infrared Spectrometersand Telescopes for the Atmosphere (CRISTA) experiment and middle atmosphere variability, J. Geophys. Res.-Atmos., 104, 16311-16325, doi:10.1029/1998JD100047, 1999.

Peter, T. and Grooß, J.-U.: Polar stratospheric clouds and sulfate aerosol particles: microphysics, denitrification and heterogeneous chemistry, in: Stratospheric Ozone Depletion and Climate Change, edited by: Müller, R., RSC Publishing, UK, 108-144, 2012.

Phillips, C.: A technique for the numerical solution of certain integral equations of the first kind, J. ACM, 9, 84-97, 1962.

Piesch, C., Gulde, T., Sartorius, C., Friedl-Vallon, F., Seefeldner, M., Wölfel, M., Blom, C. E., and Fischer, H.: Design of a MIPAS Instrument for High-Altitude Aircraft, Proc. of the 2nd Internat. Airborne Remote Sensing Conference and Exhibition, ERIM, Ann Arbor, MI, Vol. II, San Francisco, 24-27 June 1996, 199-208, 1996.

Purser, R. J. and Huang, H.-L.: Estimating effective data density in a satellite retrieval or an objective analysis, J. Appl. Meteorol., 32, 1092-1107, 1993.

Riediger, O., Volk, C. M., Strunk, M., and Schmidt, U.: HAGAR - a new in situ tracer instrument for stratospheric balloons and high altitude aircraft, Eur. Comm. Air Pollut. Res. Report, 73, 727-730, 2000.

Riese, M., Oelhaf, H., Preusse, P., Blank, J., Ern, M., Friedl-Vallon, F., Fischer, H., Guggenmoser, T., Höpfner, M., Hoor, P., Kaufmann, M., Orphal, J., Plöger, F., Spang, R., Suminska-Ebersoldt, O., Ungermann, J., Vogel, B., and Woiwode, W.: Gimballed Limb Observer for Radiance Imaging of the Atmosphere (GLORIA) scientific objectives, Atmos. Meas. Tech., 7, 1915-1928, doi:10.5194/amt-7-1915-2014, 2014.

Remedios, J. J., Leigh, R. J., Waterfall, A. M., Moore, D. P., Sembhi, H., Parkes, I., Greenhough, J., Chipperfield, M.P., and Hauglustaine, D.: MIPAS reference atmospheres and comparisons to V4.61/V4.62 MIPAS level 2 geophysical data sets, Atmos. Chem. Phys. Discuss., 7, 9973-10017, doi:10.5194/acpd-79973-2007, 2007.

Rodgers, C. D.: Inverse Methods for Atmospheric Sounding: Theory and Practice, edited by: Taylor, F. W., vol. 2 of Series on Atmospheric, Oceanic and Planetary Physics, World Scientific, 2000.

Spang, R., Remedios, J. J., and Barkley, M. P.: Colour indices for the detection and differentiation of cloud types in infra-red limb emission spectra, Adv. Space Res., 33, 1041-1047, 2004.

Spang, R., Hoffmann, L., Kullmann, A., Olschewski, F., Preusse, P., Knieling, P., Schroeder, S., Stroh, F., Weigel, K., and Riese, M.: High resolution limb observations of clouds by the CRISTA-NF experiment during the SCOUT-O3 tropical aircraft campaign, Adv. Space Res., 42, 1765-1775, doi:10.1016/j.asr.2007.09.036, 2008.

Stefanutti, L., Sokolov, L., Balestri, S., MacKenzie, A. R., and Khattatov, V.: The M-55 Geophysica as a platform for the air- 
borne polar experiment, J. Atmos. Ocean. Tech., 16, 1303-1312, doi:10.1175/1520-0426(1999)016<1303:tmgaap >2.0.co;2, 1999.

Stiller, G. P., von Clarmann, T., Funke, B., Glatthor, N., Hase, F., Höpfner, M., and Linden, A.: Sensitivity of trace gas abundances retrievals from infrared limb emission spectra to simplifying approximations in radiative transfer modelling, J. Quant. Spectrosc. Ra., 72, 249-280, doi:10.1016/S0022-4073(01)00123-6, 2002.

Tikhonov, A.: On the solution of incorrectly stated problems and a method of regularisation, Dokl. Acad. Nauk SSSR, 151, 501504, 1963.

Ungermann, J., Kalicinsky, C., Olschewski, F., Knieling, P., Hoffmann, L., Blank, J., Woiwode, W., Oelhaf, H., Hösen, E., Volk, C. M., Ulanovsky, A., Ravegnani, F., Weigel, K., Stroh, F., and Riese, M.: CRISTA-NF measurements with unprecedented vertical resolution during the RECONCILE aircraft campaign, Atmos. Meas. Tech., 5, 1173-1191, doi:10.5194/amt-5-1173-2012, 2012.

Ungermann, J., Pan, L. L., Kalicinsky, C., Olschewski, F., Knieling, P., Blank, J., Weigel, K., Guggenmoser, T., Stroh, F., Hoffmann, L., and Riese, M.: Filamentary structure in chemical tracer distributions near the subtropical jet following a wave breaking event, Atmos. Chem. Phys., 13, 10517-10534, doi:10.5194/acp13-10517-2013, 2013.

von Clarmann, T., Glatthor, N., Grabowski, U., Höpfner, M., Kellmann, S., Kiefer, M., Linden, A., Mengistu Tsidu, G., Milz, M., Steck, T., Stiller, G. P., Wang, D. Y., Fischer, H., Funke, B., Gil-López, S., and López-Puertas, M.: Retrieval of temperature and tangent altitude pointing from limb emission spectra recorded from space by the Michelson Interferometer for Passive Atmospheric Sounding (MIPAS), J. Geophys. Res., 108, 4736, doi:10.1029/2003JD003602, 2003.

von Hobe, M., Bekki, S., Borrmann, S., Cairo, F., D’Amato, F., Di Donfrancesco, G., Dörnbrack, A., Ebersoldt, A., Ebert, M., Emde, C., Engel, I., Ern, M., Frey, W., Genco, S., Griessbach, S., Grooß, J.-U., Gulde, T., Günther, G., Hösen, E., Hoffmann, L., Homonnai, V., Hoyle, C. R., Isaksen, I. S. A., Jackson, D. R., Jánosi, I. M., Jones, R. L., Kandler, K., Kalicinsky, C., Keil, A., Khaykin, S. M., Khosrawi, F., Kivi, R., Kuttippurath, J., Laube, J. C., Lefèvre, F., Lehmann, R., Ludmann, S., Luo, B. P., Marchand, M., Meyer, J., Mitev, V., Molleker, S., Müller, R., Oelhaf, H., Olschewski, F., Orsolini, Y., Peter, T., Pfeilsticker, K., Piesch, C., Pitts, M. C., Poole, L. R., Pope, F. D., Ravegnani, F., Rex, M., Riese, M., Röckmann, T., Rognerud, B., Roiger, A., Rolf, C., Santee, M. L., Scheibe, M., Schiller, C., Schlager, H., Siciliani de Cumis, M., Sitnikov, N., Søvde, O. A., Spang, R., Spelten, N., Stordal, F., Sumińska-Ebersoldt, O., Ulanovski, A., Ungermann, J., Viciani, S., Volk, C. M., vom Scheidt, M., von der Gathen, P., Walker, K., Wegner, T., Weigel, R., Weinbruch, S., Wetzel, G., Wienhold, F. G., Wohltmann, I., Woiwode, W., Young, I. A. K., Yushkov, V., Zobrist, B., and Stroh, F.: Reconciliation of essential process parameters for an enhanced predictability of Arctic stratospheric ozone loss and its climate interactions (RECONCILE): activities and results, Atmos. Chem. Phys., 13, 92339268, doi:10.5194/acp-13-9233-2013, 2013.
Werner, A., Volk, C. M., Ivanova, E. V., Wetter, T., Schiller, C., Schlager, H., and Konopka, P.: Quantifying transport into the Arctic lowermost stratosphere, Atmos. Chem. Phys., 10, 1162311639, doi:10.5194/acp-10-11623-2010, 2010.

Woiwode, W., Oelhaf, H., Gulde, T., Piesch, C., Maucher, G., Ebersoldt, A., Keim, C., Höpfner, M., Khaykin, S., Ravegnani, F., Ulanovsky, A. E., Volk, C. M., Hösen, E., Dörnbrack, A., Ungermann, J., Kalicinsky, C., and Orphal, J.: MIPAS-STR measurements in the Arctic UTLS in winter/spring 2010: instrument characterization, retrieval and validation, Atmos. Meas. Tech., 5, 1205-1228, doi:10.5194/amt-5-1205-2012, 2012.

Zöger, M., Afchine, A., Eicke, N., Gerhards, M. T., Klein, E., McKenna, D. S., Morschel, U., Schmidt, U., Tan, V., Tuitjer, F., Woyke, T., and Schiller, C.: Fast in situ stratospheric hygrometers: a new family of balloon-borne and airborne Lyman alpha photofragment fluorescence hygrometers, J. Geophys. Res., 104, 1807-1816, 1999. 